\title{
A Novel Bibenzyl Compound (20C) Protects Mice from 1-Methyl-4-Phenyl-1,2,3,6-Tetrahydropyridine/Probenecid Toxicity by Regulating the $\alpha$-Synuclein-Related Inflammatory Response
}

\author{
Qui-Shuang Zhang, Yang Heng, Ying Chen, Piao Luo, Lu Wen, Zhao Zhang, \\ Yu-He Yuan, and Nai-Hong Chen
}

State Key Laboratory of Bioactive Substances and Functions of Natural Medicines, Institute of Materia Medica and Neuroscience Center, Chinese Academy of Medical Sciences and Peking Union Medical College, Beijing, China (Q.-S.Z., Y.H., Y.C., L.W., Z.Z., Y.-H.Y., N.-H.C.); and College of Pharmacy, Hunan University of Chinese Medicine, Changsha, Hunan, China (P.L., N.-H.C.)

Received July 16, 2017; accepted September 12, 2017

\begin{abstract}
The novel bibenzyl compound 2-[4-hydroxy-3-(4- hydroxyphenyl) benzyl]-4-(4- hydroxyphenyl) phenol (20C) plays a neuroprotective role in vitro, but its effects in vivo have not yet been elucidated. In this study, we estimated the efficacy of $20 \mathrm{C}$ in vivo using a 1-methyl-4-phenyl-1,2,3,6-tetrahydropyridine/probenecid (MPTP/p) mouse model from behavior, dopamine, and neuron and then the possible mechanisms for these effects were further investigated. The experimental results showed that $20 \mathrm{C}$ improved behavioral deficits, attenuated dopamine depletion, reduced
\end{abstract}

dopaminergic neuron loss, protected the blood-brain barrier (BBB) structure, ameliorated $\alpha$-synuclein dysfunction, suppressed glial activation, and regulated both nuclear factor $-\kappa \mathrm{B}$ (NF- $\kappa \mathrm{B}$ ) signaling and the NOD-like receptor protein (NLRP) 3 inflammasome pathway. Our results indicated that $20 \mathrm{C}$ may prevent neurodegeneration in the MPTP/p mouse model by targeting $\alpha$-synuclein and regulating $\alpha$-synuclein-related inflammatory responses, including BBB damage, glial activation, NF- $\kappa \mathrm{B}$ signaling, and the NLRP3 inflammasome pathway.

\section{Introduction}

Parkinson's disease (PD) is well known for motor disorders such as resting tremor, bradykinesia, muscle rigidity, and postural instability and affects $1 \%$ of the population aged $>60$ years (Connolly and Lang, 2014). Typical characteristics of PD include progressive loss of dopaminergic cells in the substantia nigra pars compacta (SNpc), dopamine (DA) depletion in the striatum, and formation of Lewy bodies (LBs) (Connolly and Lang, 2014). The current therapy for PD relies on DA supplementation from its precursor, L-dopa, which can quickly improve behavioral symptoms; however, long-term treatment with L-dopa may induce considerable adverse effects (Connolly and Lang, 2014). Therefore, it is necessary to develop new efficient candidates based on PD pathogenesis. The exact etiology for PD remains unknown, and neurotoxin-based

This work was supported by the National Natural Science Foundation of China [Grants 81773925, U1402221, 81573640, and 81603316], the Beijing Natural Science Foundation [Grant 7161011], the Chinese Academy of Medical Sciences Innovation Fund for Medical Sciences [Grant 2016-I2M-1-004], the Key Research and Development Project of Hunan Province [Grant 2015SK2029-1], and the Scientific Research Foundation of the Higher Education Institutions of Hunan Province [Grant 15K091].

https://doi.org/10.1124/jpet.117.244020. experimental models of PD have been widely used to mimic the clinical parkinsonian features. To some extent, these models conform to a hypothesis of pathogenesis that involves $\alpha$-synuclein dysfunction, inflammation, and oxidative stress (Dauer and Przedborski, 2003; Zhang et al., 2017a). Among these models, the 1-methyl-4-phenyl-1,2,3,6-tetrahydropyridine (MPTP)-induced animal model is widely used in the study of PD. MPTP can cross the blood-brain barrier (BBB) and can be metabolized into its toxic form (1-methyl-4-phenylpyridinium ion) by monoamine oxidase $\mathrm{B}$ in the glia; 1-methyl-4phenylpyridinium ion can then be transferred to dopaminergic neurons via DA transporters to induce cell death (Dauer and Przedborski, 2003). The chronic 1-methyl-4-phenyl-1,2,3,6tetrahydropyridine/probenecid (MPTP/p) model is more commonly used to mimic the neurodegenerative process of PD than other models. In the MPTP/p model, probenecid is applied to enhance the toxicity of MPTP by suppressing its clearance (Bové and Perier, 2012). The specific monoamine oxidase B inhibitor, selegiline, is a reliable reference for efficacy evaluation in this model.

The traditional herbal medicine, Gastrodia elata (Tianma), has been used for centuries to treat headache, dizziness, epilepsy, rheumatism, neuralgia, paralysis, and other

ABBREVIATIONS: BBB, blood-brain barrier; CNS, central nervous system; DA, dopamine; DMSO, dimethylsulfoxide; GSDMD, gasdermin D; IL, interleukin; INF, interferon; LB, Lewy body; MPTP, 1-methyl-4-phenyl-1,2,3,6-tetrahydropyridine; MPTP/p, 1-methyl-4-phenyl-1,2,3,6-tetrahydropyridine/probenecid; $\mathrm{NF}-\kappa \mathrm{B}$, nuclear factor- $\kappa \mathrm{B}$; NLRP, NOD-like receptor protein; PD, Parkinson's disease; SNpc, substantia nigra pars compact; TH, tyrosine hydroxylase; TLR, Toll-like receptor. 
A<smiles>Oc1ccc(Cc2ccc(O)c(Cc3ccc(O)c(Cc4ccc(O)cc4)c3)c2)cc1</smiles>

B

\begin{tabular}{lllllll}
\hline Groups & Ctrl & Model & 20C & & & Sele \\
\hline p.o. & CMCNa & CMCNa & $20 \mathrm{C}(25 \mathrm{mg} / \mathrm{kg}$ & $50 \mathrm{mg} / \mathrm{kg}$ & $100 \mathrm{mg} / \mathrm{kg})$ & selegiline $(3 \mathrm{mg} / \mathrm{kg})$ \\
i.p. & probenecid & probenecid & probenecid & probenecid & probenecid & probenecid \\
s.c. & NS & MPTP & MPTP & MPTP & MPTP & MPTP \\
\hline
\end{tabular}

C administration of $20 \mathrm{C}$ or selegiline every day

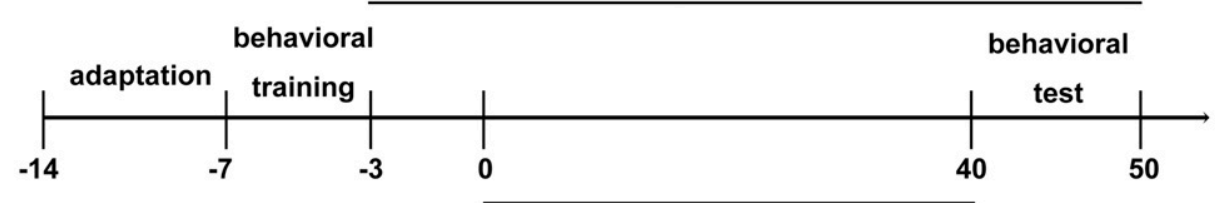

MPTP/p treatment,

once every other 3 days, for ten times

D

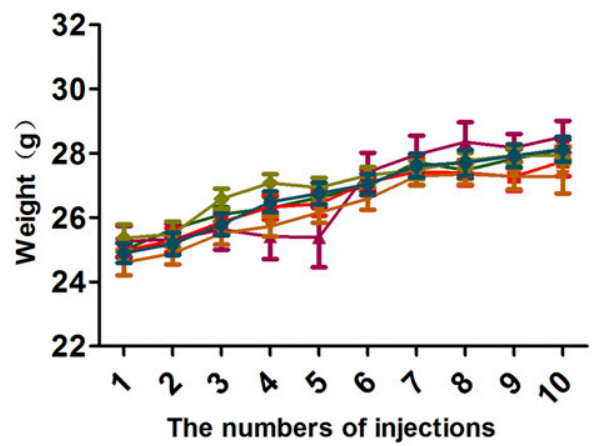

E

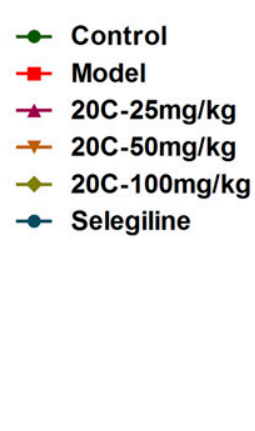

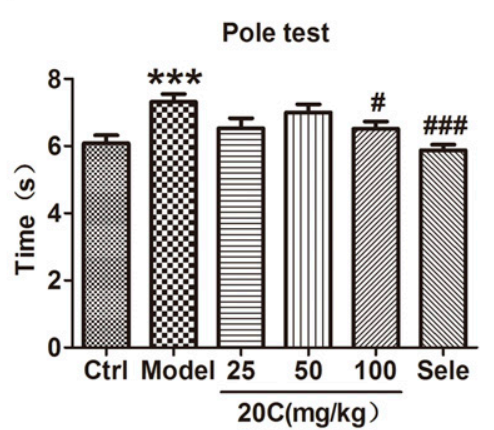

Fig. 1. Experimental procedure, body weight, and pole test. (A) Chemical structure of 20C. (B) Treatment of each group. (C) Schedule for this study. (D) There was no difference in body weight among groups. (E) 20C could improve motor impairment in the pole test. Data are presented as means \pm S.E.M. $(n>10)$. One-way analysis of variance was followed by Duncan's post hoc tests. ${ }^{* * *} P<0.001 ;{ }^{\#} P<0.05$; ${ }^{\# \# \#} P<0.001$ vs. the control group. CMCNa, sodium carboxymethylcellulose; Ctrl, control; NS, normal saline.

neuralgic disorders (Ojemann et al., 2006). Moreover, Tianma (G. elata) Gouteng Yin has been commonly prescribed in China to treat parkinsonian symptoms including tremor and paralysis (Liu et al., 2015). 20C is a novel bibenzyl compound derived from G. elata, and its neuroprotective effect in vitro has been described; 20C has been found to promote antiapoptosis and oxidation resistance, reduce $\alpha$-synuclein accumulation, and inhibit endoplasmic reticulum stress (Huang et al., 2016; Mou et al., 2016; Zhang et al., 2017c). In this study, we investigated its protective effect and associated mechanism in vivo using the MPTP/p mouse model.

\section{Materials and Methods}

Reagents and Animals. The 20C compound (Fig. 1A) was provided by the Department of Chemosynthesis, Institute of Materia Medica, Chinese Academy of Medical Sciences and Peking Union Medical College (Beijing, China). Selegiline hydrochloride
(Orion Co., Espoo, Finland) was purchased from Beijing Hospital. MPTP hydrochloride and probenecid were obtained from SigmaAldrich (St. Louis, MO). Male C57BL/6 mice (8 weeks, 22-25 g; Charles River Co., Beijing, China) were used in the experiments. All mice were housed on a 12-hour light/dark cycle at a temperature of $22 \pm 1^{\circ} \mathrm{C}$, with free access to food and water. All animal experiments were approved by the Animal Care Committee of the Chinese Academy of Medical Sciences, according to the principles established by the National Institutes of Health for the care and use of laboratory animals.

Experimental Procedures. After 1 week of adaptation, all animals were randomly divided into six groups (Fig. 1B): the control group (equivoluminal water), the model group (equivoluminal water), the $20 \mathrm{C}$ groups $(25,50$, and $100 \mathrm{mg} / \mathrm{kg})$, and the selegiline group $(3 \mathrm{mg} / \mathrm{kg})$. Then they were trained on the pole test. The chronic mouse model was established as follows (Fig. 1C). Mice received an intraperitoneal injection of probenecid $(250 \mathrm{mg} / \mathrm{kg}$ body weight, dissolved in $0.1 \mathrm{M} \mathrm{NaOH}$-Tris-HCl, $\mathrm{pH} 7.4$ ); 1 hour later, they were subcutaneously injected with MPTP-HCl $(25 \mathrm{mg} / \mathrm{kg}$, dissolved in $0.9 \%$ 
saline). This process of MPTP/p injection was conducted every 4 days and a total of 10 doses were administered. 20C, selegiline, or equivoluminal water was administered 3 days before starting this MPTP/p scheme, and the administration continued for 53 days. After the mice completed the MPTP/p administration protocol, the pole test was implemented to assess their behavioral performance. Ten days after the last injection, mice were euthanized for further study.

Behavioral Testing. Behavioral performance was evaluated with the pole test as described in our previous work (Heng et al., 2016). The test's objective is to measure the time required for the mice to climb down from the top of the pole. After adaptation, the mice were pretrained on the pole apparatus for three sessions to ensure that all animals used for experiments would climb down as soon as they were put on the ball. During testing, the time required to climb down was recorded.

Tissue Preparation. For the biochemical analysis, mice were deeply anesthetized with chloral hydrate $(400 \mathrm{mg} / \mathrm{kg}$, i.p.), and brain tissues were rapidly isolated and stored at $-80^{\circ} \mathrm{C}$. For the histologic analysis after anesthesia, mice were perfused intracardially with $0.1 \mathrm{M}$ phosphate-buffered saline and $4 \%$ paraformaldehyde. The mouse brains were then collected and preserved in $4 \%$ paraformaldehyde. Before detection, the brain samples were dehydrated with graded sucrose $(10 \%, 20 \%$, and $30 \%)$, and then coronal sections $(20 \mu \mathrm{m})$ containing the striatum region or the SNpc region were dissected on a cryostat (CM3050S; Leica, Solms, Germany). Finally, these sections were laid on coated slides for detection.

High-Performance Liquid Chromatography. Striatal tissues were diluted 10 -fold (w/v) with ice-cold solution A (which contained $0.6 \mathrm{M} \mathrm{HClO}_{4}$ and $375 \mathrm{ng} / \mathrm{ml}$ isoprenaline), homogenized, and then centrifuged at $20,000 \mathrm{~g}$ at $4^{\circ} \mathrm{C}$ for 20 minutes. The supernatant was collected and mixed with a half volume of solution $\mathrm{B}$ (which contained $20 \mathrm{mM}$ potassium citrate, $300 \mathrm{mM} \mathrm{K} \mathrm{HPO}_{4}$, and $2 \mathrm{mM}$ EDTA $2 \mathrm{Na}$ ) and was centrifuged again, filtered, and injected into the highperformance liquid chromatography system (Waters e2695; Waters, Milford, MA) equipped with a C18 column $(4.6 \mathrm{~mm}, 150 \mathrm{~mm}$, Atlantis T3; Waters) and an electrochemical detector (Waters 2465; Waters). The mobile phase ( $100 \mathrm{mM}$ sodium acetate anhydrous, $85 \mathrm{mM}$ citric acid, $0.5 \mathrm{mM}$ 1-octanesulfonic acid sodium, $0.2 \mathrm{mM}$ EDTA $2 \mathrm{Na}$, and $15 \%$ methanol, $\mathrm{pH} 3.68$ ) was pumped at a rate of $1 \mathrm{ml} / \mathrm{min}$. The detection potential was set at $760 \mathrm{mV}$ versus $\mathrm{Ag} / \mathrm{AgCl}$ and the assay sensitivity was set at a scale of $50 \mathrm{nA}$.

Western Blot Analysis. After the striatal tissue was disrupted and centrifuged, the supernatant was collected, measured using a BCA (Bicinchoninic acid) kit and denatured in loading buffer. Samples of equal protein content were separated by $15 \%$ PAGE and transferred to polyvinylidene difluoride membranes (Millipore, Milford, MA). After the membranes were blocked with $3 \%$ bovine serum albumin (SigmaAldrich), they were incubated with primary antibodies, including anti- $\beta$-actin (1:5000; Sigma-Aldrich), anti-tyrosine hydroxylase (TH) (1:500; Santa Cruz, Santa Cruz, CA), anti- $\alpha$-synuclein (C20) (1:500; Santa Cruz), anti-5G4 (1:1000; Millipore), antinitrated Tyr125/Tyr133 $\alpha$-synuclein (1:1000; Thermo, Waltham, MA), antiphosphorylated $\alpha$-synuclein (1:1000; Abcam, Cambridge, MA), anti-Toll-like receptor (TLR) 4 (1:250; Santa Cruz), anti-nuclear factor- $\kappa \mathrm{B}(\mathrm{NF}-\kappa \mathrm{B})$ (1:500; Santa Cruz), anti-NOD-like receptor protein (NRLP) 3 (1:1000; Abcam), anti-caspase-1 (1:1000; Abcam), anti-interleukin (IL)-1 $\beta$ (1: 250; Santa Cruz), and anti-gasdermin D (GSDMD) (1:1000; SigmaAldrich). After washing, the membranes were incubated with horseradish peroxidase-conjugated secondary antibody (1:5000; KPL, Gaithersburg, MD) for 2 hours. The blots were detected by enhanced chemiluminescence plus the LMAX detection system (Molecular Devices, Sunnyvale, CA) and were quantified with image analysis software (Quantity One, Toyobo, Japan).

Electron Microscopy Analysis. Anesthetized mice were perfused and fixed with $4 \%$ paraformaldehyde, and cubic sections $\left(1 \mathrm{~mm}^{3}\right)$ of the SNpc region were dissected and fixed in $2.5 \%$ glutaraldehyde for 2 hours. After washing, these samples were dehydrated in graded alcohol solution and embedded in Epon resin. The regions of interest were then cut into ultrathin sections, which were then doubly stained with uranyl acetate and lead citrate. Finally, the ultrastructure of the SNpc region was observed under a transmission electron microscope (H-7650; Hitachi, Tokyo, Japan).

Immunohistochemistry and Immunofluorescence. The brain slides were boiled in $0.01 \mathrm{M}$ citrate buffer solution for antigen retrieval and were incubated with $1 \%$ Triton X-100 to increase antibody penetration. Hydrogen peroxide $(3 \%)$ was then used to eliminate endogenous peroxidase activity. After the sections were blocked with $5 \%$ bovine serum albumin, they were incubated with anti-TH (1:100; Santa Cruz), anti-glial fibrillary acidic protein (1:500; Dako, Glostrup, Denmark), or anti-ionized calcium binding adapter molecule-1 (1:100; Wako, Osaka, Japan) overnight or with anti-5G4 (1:200; Millipore), antinitrated Tyr125/Tyr133 $\alpha$-synuclein (1:200; Thermo), anticaspase-1 p10 (1:50; Santa Cruz), anti-IL-1 $\beta$ (1: 50; Santa Cruz), anti-8-hydroxyguanosine (1:100; Abcam), and anti-GSDMD (1:100; Sigma-Aldrich). After washing, sections were incubated with horseradish peroxidase-conjugated secondary antibody (1:200; KPL) or Alexa Fluor-conjugated secondary antibody (1:500; Invitrogen, Camarillo, CA). For immunohistochemistry, sections were detected with 3,3-diaminobenzidine and captured with an Olympus BA51 photomicroscope (Tokyo, Japan). For immunofluorescence, images were acquired with a fluorescence microscope (Nikon, Tokyo, Japan) or a laser scanning confocal microscope (Leica TCS SP2). Finally, Image Pro Plus 6.0 software (Media Cybernetics, Rockville, MD) was used for cell counting at $\times 100$ magnification.

Statistical Analysis. Experimental data were statistically analyzed by one-way analysis of variance followed by Duncan's test. All values are displayed as means \pm S.E.M., and a $P$ value of $<0.05$ was considered statistically significant.

\section{Results}

Body Weight, Behavior Assessment, and Neurotransmission. Body weight was not significantly affected by $\mathrm{MPTP} / \mathrm{p}$ treatment or $20 \mathrm{C}$ administration (Fig. 1D). The behavioral assessment with the pole test showed that MPTP/p-treated mice took longer to complete the test $(P<0.001)$, and 20C $(100 \mathrm{mg} / \mathrm{kg}, P<0.05)$ or selegiline $(P<0.001)$ had an improving effect (Fig. 1E).

Protective Effect of 20C on the Nigrostriatal Dopaminergic System. DA in the striatum was analyzed with high-performance liquid chromatography (Fig. 2, A-C). MPTP/p caused a marked reduction in DA, whereas $20 \mathrm{C}$ (50 and $100 \mathrm{mg} / \mathrm{kg}, P<0.05)$ and selegiline $(P<0.001)$ significantly increased DA content. Moreover, DA turnover occurred in MPTP/p-treated mice; that is, the value of dihydroxyphenylacetic acid/DA $(P<0.001)$ and homovanillic acid/DA $(P<0.001)$ increased significantly after MPTP/p treatment. $20 \mathrm{C}(50 \mathrm{mg} / \mathrm{kg}, P<0.01$ for dihydroxyphenylacetic acid/DA, $P<0.05$ for homovanillic acid/DA; $100 \mathrm{mg} / \mathrm{kg}, P<$ $0.05)$ and selegiline $(P<0.01)$ had an improving effect. TH, the rate-limiting enzyme in DA synthesis, is highly expressed in dopaminergic neurons; therefore, $\mathrm{TH}^{+}$cells are considered to represent dopaminergic neurons. Immunohistochemical staining and Western blot bands showed that the dopaminergic cells in the SNpc and the terminals in the striatum were markedly lost. 20C and selegiline had a protective effect on both dopaminergic cells and terminals (Fig. 2, D-H).

Protective Effect of $20 \mathrm{C}$ on the Ultrastructure of the SNpc Region. Ultrastructural changes in the SNpc region were further studied with transmission electron microscopy (Fig. 2, I-K). As shown in the images, the normal neuron had a low electron density and a round smooth contour, and the 
A

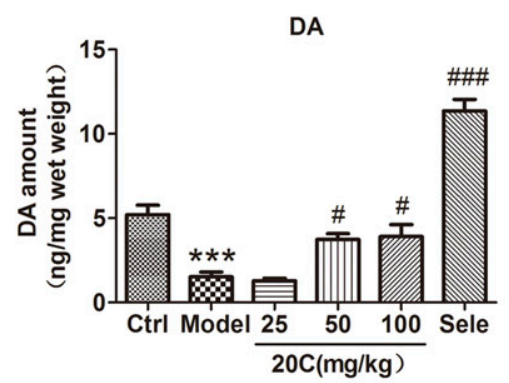

D

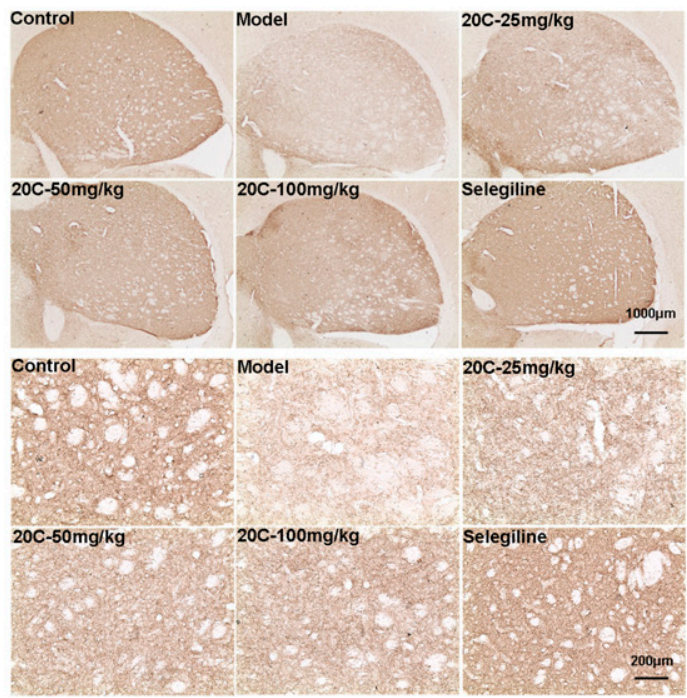

DOPAC/DA

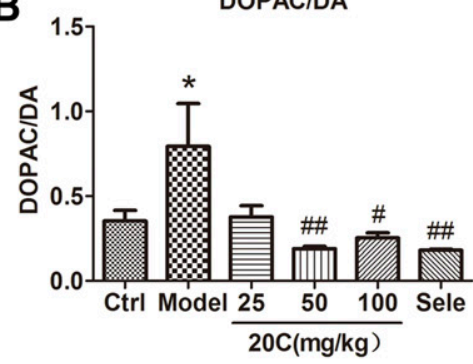

HVADA

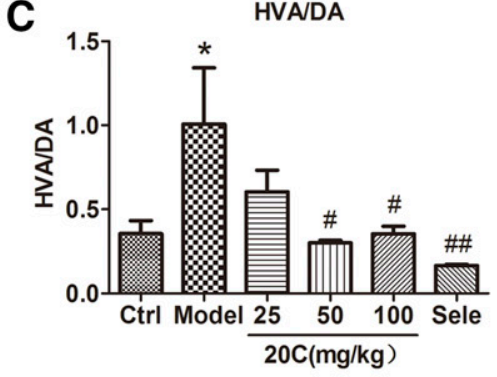

E
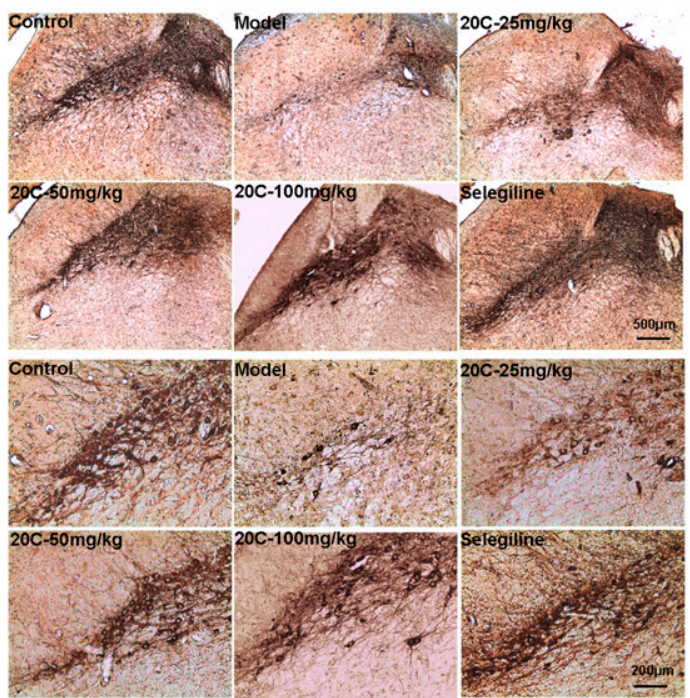

H
$\mathbf{F}$ TH Ctrl Model $25 \quad 50 \quad 100$ Sele

$\beta$-actin

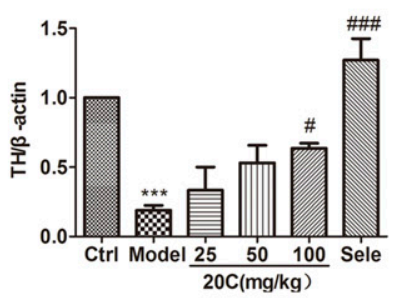

I

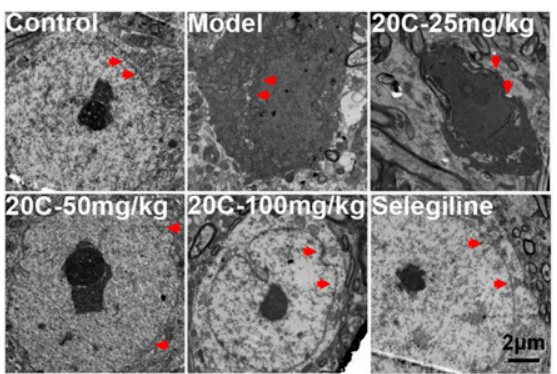

G

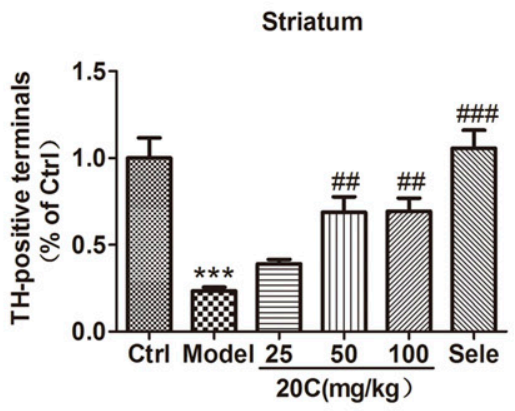

J

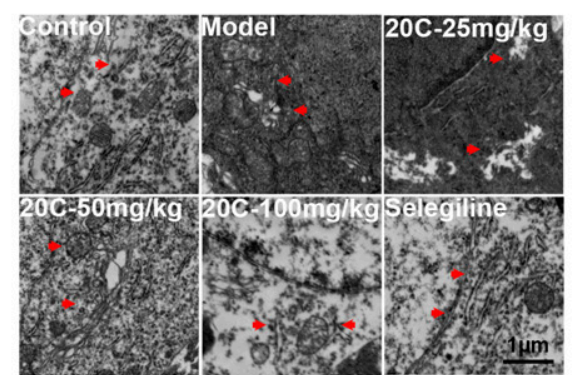

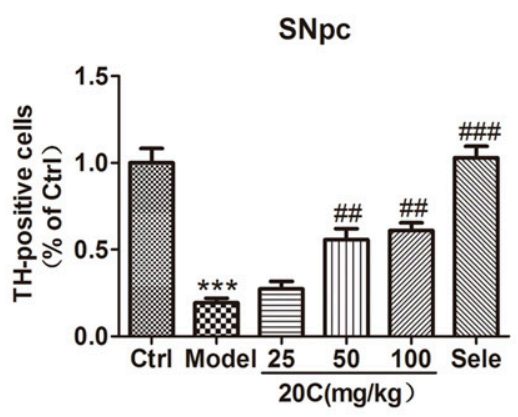

K

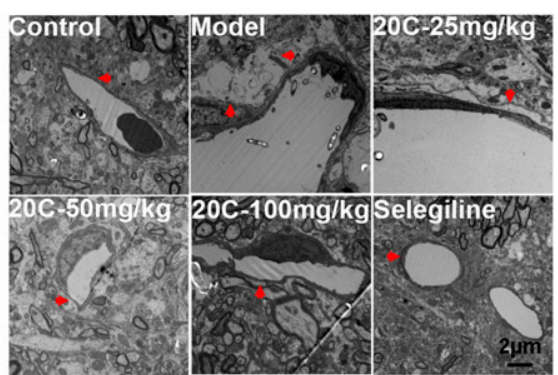

Fig. 2. $20 \mathrm{C}$ had a protective effect on the nigrostriatal system. (A-C) $20 \mathrm{C}$ could attenuate DA depletion and DA turnover. (D-H) $20 \mathrm{C}$ could reduce the loss of dopaminergic cells in the SNpc and terminals in the striatum. (D and E) Immunohistochemical staining of TH in the nigrostriatal system. (F) Western blot results for $\mathrm{TH}$ protein levels in the striatum. (G and $\mathrm{H}$ ) Quantitative analysis of $\mathrm{TH}^{+}$cells in the SNpc and striatum. (I-K) 20C could ameliorate ultrastructure destruction in the SNpc region. As indicated by the red arrows, the model neuron is severely damaged with heavy electron density (I) and swollen organelles (J), and the structure of the BBB is seriously destroyed (K), which could be improved by $20 \mathrm{C}$ and selegiline. Data are presented as means \pm S.E.M. $(n=3)$. One-way analysis of variance was followed by Duncan's post hoc tests. $* P<0.05 ; * * * P<0.001$ vs. the control group; ${ }^{\#} P<0.05 ;{ }^{\# \#} P<0.01 ;{ }^{\# \#} P<0.001$ vs. the model group. Ctrl, control; DOPAC, dihydroxyphenylacetic acid; HVA, homovanillic acid. 

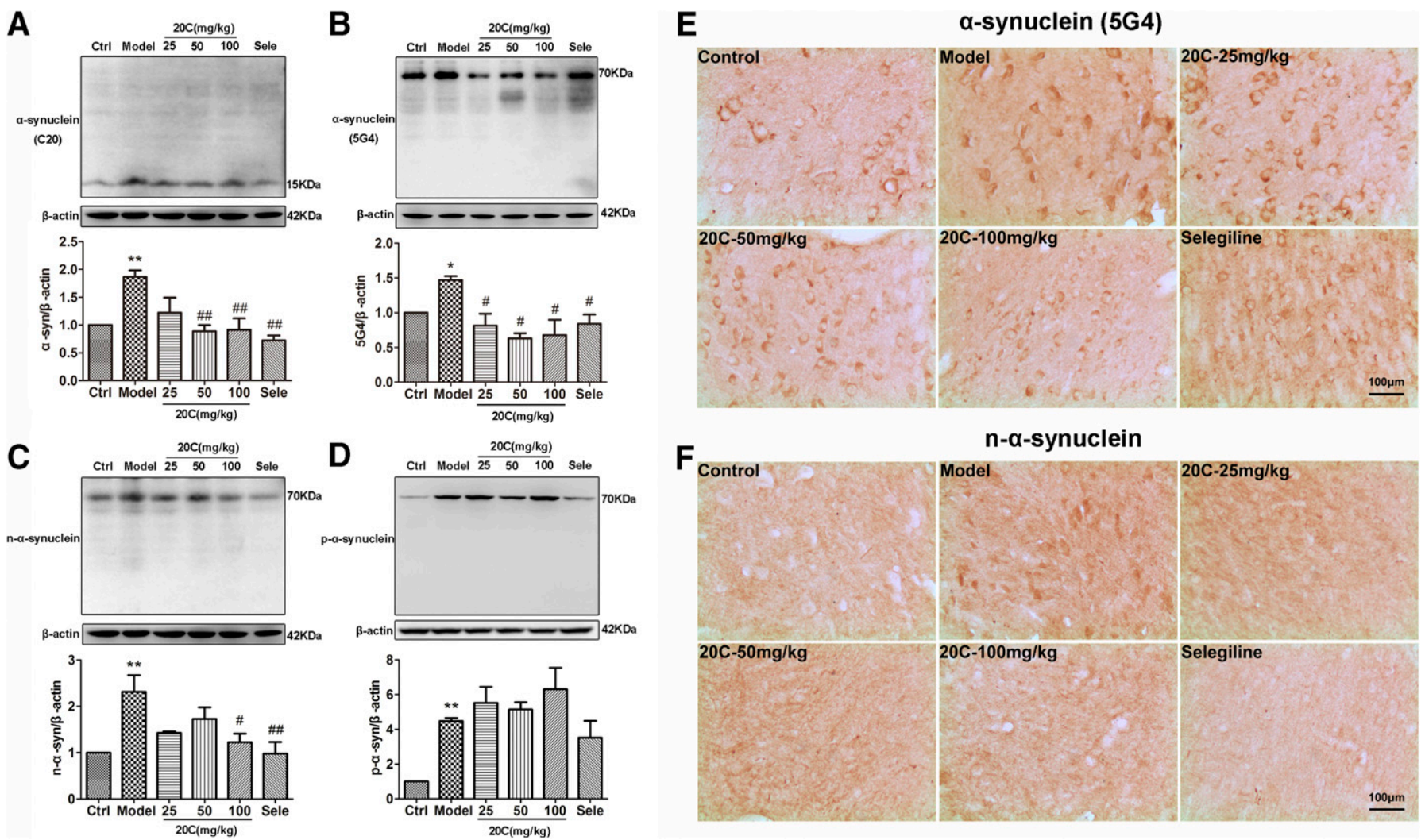

Fig. 3. 20C could ameliorate MPTP/p-induced $\alpha$-synuclein dysfunction. (A-D) Western blot results for $\alpha$-synuclein (C20), aggregated $\alpha$-synuclein (5G4), nitrated Tyr125/Tyr133 $\alpha$-synuclein, and phosphorylated Ser129 $\alpha$-synuclein. (E and F) Immunohistochemical results for aggregated $\alpha$-synuclein (5G4) and nitrated Tyr125/Tyr133 $\alpha$-synuclein. Data are presented as means \pm S.E.M. $(n=3)$. One-way analysis of variance was followed by Duncan's post hoc tests. ${ }^{*} P<0.05 ;{ }^{* *} P<0.01 \mathrm{v}$. the control group; ${ }^{\sharp} P<0.05 ;{ }^{\# \#} P<0.01$ vs. the model group. Ctrl, control.

organelle structure was relatively integrated. After MPTP/p treatment, the neuron became irregular, lost its shape, and obtained darker electron density, and the organelles were swollen and incomplete. $20 \mathrm{C}$ and selegiline ameliorated MPTP/p-induced neuronal damage (Fig. 2, I and J). Moreover, the BBB in the SNpc region was severely injured by MPTP/p, and $20 \mathrm{C}$ and selegiline could alleviate the injury (Fig. 2K).

Protective Effect of 20C on MPTP-Induced $\alpha$-Synuclein Abnormality. In this study, we detected a significant increase in different $\alpha$-synuclein forms after MPTP/p treatment (Fig. 3, A-D), such as the monomeric $\alpha$-synuclein (C20) $(P<0.01)$, disease-related aggregate (5G4) $(P<0.05)$, nitrated $\alpha$-synuclein $(P<0.01)$, and phosphorylated $\alpha$-synuclein $(P<0.01)$. Regarding increased monomeric $\alpha$-synuclein (C20), 20C (50 and $100 \mathrm{mg} / \mathrm{kg}$, $P<0.01)$ and selegiline $(P<0.01)$ had a significant suppressive effect. For the abnormal $\alpha$-synuclein aggregate (5G4), 20C (25, 50 , and $100 \mathrm{mg} / \mathrm{kg}, P<0.05)$ and selegiline $(P<0.05)$ had a significant relieving effect. For nitrated $\alpha$-synuclein, 20C $(100 \mathrm{mg} / \mathrm{kg}, P<0.05)$ and selegiline $(P<0.01)$ had a significant ameliorating effect. However, for phosphorylated $\alpha$-synuclein, there was no evident reduction in the $20 \mathrm{C}$ groups and the selegiline group. Immunohistochemical staining (Fig. 3, E and F) for the abnormal $\alpha$-synuclein aggregate (5G4) and nitrated $\alpha$-synuclein showed the same trends with the Western blot analysis.

Suppression of 20C on MPTP-Induced Activation of Astrocytes and Microglia. Astrocytes and microglia are the cells that are principally involved in brain immunity. Our immunohistochemical results (Fig. 4) showed that the amount of both astrocytes and microglia in the $\mathrm{SNpc}$ region were largely increased and activated by MPTP/p treatment. 20C (50 and $100 \mathrm{mg} / \mathrm{kg}$ ) and selegiline could obviously suppress this activation $(P<0.01$ for astrocytes, $P<0.05$ for microglia).

Regulation of 20C on MPTP-Induced NF- $\kappa$ B Signaling and the NLRP3 Inflammasome Pathway. Our results showed (Fig. 5, A-D) that MPTP/p could significantly increase the protein content of TLR4 $(P<0.01)$ (Fig. 5, A and B), NF- $\kappa \mathrm{B}(P<0.001)$ (Fig. 5, A and C), and NLRP3 $(P<0.01)$ (Fig. 5, A and D). Given that the activation of NLRP3 can lead to the cleavage of procaspase- $1(45 \mathrm{kDa})$ into $\mathrm{p} 20$ and $\mathrm{p} 10$, we detected the protein content of procaspase- 1 ( $45 \mathrm{kDa}$ ) (Fig. 5, A and E), p20 (Fig. 5, A and F), and p10 (Fig. 5G) with Western blot analysis or immunohistochemistry. Our results showed that MPTP/p led to a significant increase of caspase-1 (p20) and caspase-1 (p10). Activated caspase-1 can further shear and activate pro-IL-1 $\beta(35 \mathrm{kDa})$ and GSDMD. For IL- $1 \beta$, the Western blot results showed that MPTP/p could apparently increase IL- $1 \beta$ content $(P<0.05$ for $35 \mathrm{kDa}, P<0.01$ for $15 \mathrm{kDa}$ ) (Fig. 5, H-J), and the immunohistochemical results described the same trend (Fig. 5K). Inflammation is often accompanied by oxidative stress; we found that the model substantia nigra region experienced serious oxidative stress damage, as evident by heavily increased 8-hydroxyguanosine staining (Fig. $5 \mathrm{M})$. GSDMD $(55 \mathrm{kDa})$ is a key protein that mediates inflammatory death after shearing, and laser scanning confocal microscopy results showed that GSDMD was present in the dopaminergic neurons (Fig. 5L). Results from the Western blot analysis showed that the protein content of $55 \mathrm{kDa}$ 
A

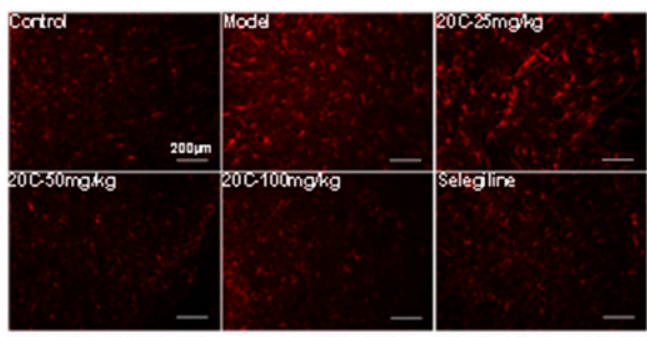

C

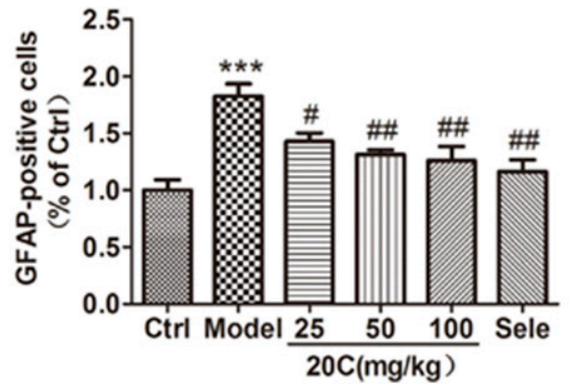

B

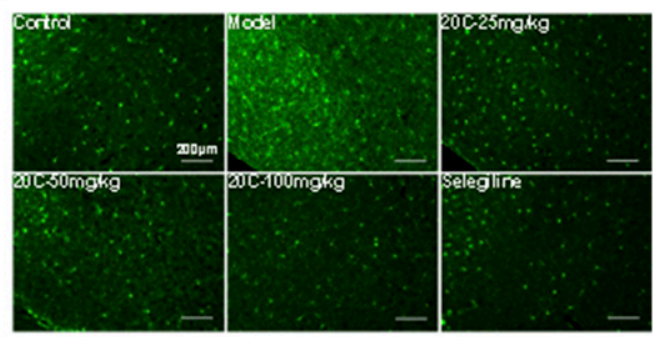

D

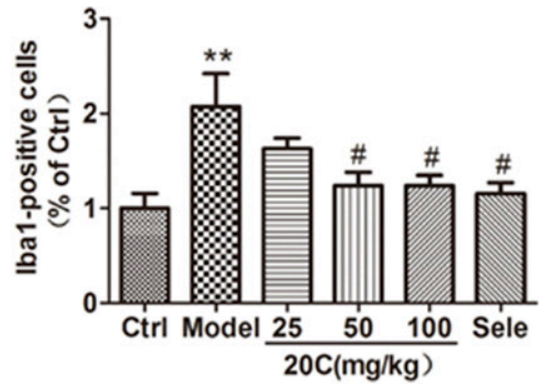

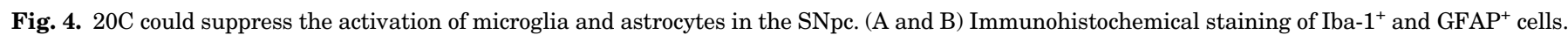

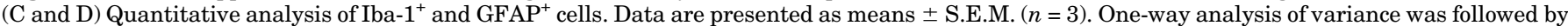

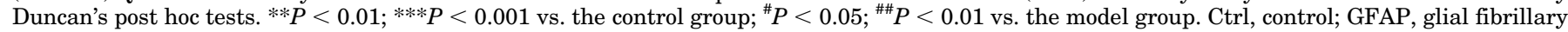
acidic protein; IBA-1, ionized calcium binding adapter molecule-1.

GSDMD was significantly decreased $(P<0.01)$ after MPTP/p treatment (Fig. 5N), which implied the activation of GSDMD. $20 \mathrm{C}$ and selegiline had a regulatory effect on these changes.

\section{Discussion}

In this study, we found that $20 \mathrm{C}$ played a protective role in the nigrostriatal system by reducing $\alpha$-synuclein dysfunction and regulating $\alpha$-synuclein-related inflammatory responses, including BBB damage, glial activation, $\mathrm{NF}-\kappa \mathrm{B}$ signaling, and the NLRP3 inflammasome pathway (Fig. 6).

$\alpha$-Synuclein, a key factor for PD-related degeneration, is highly expressed throughout the brain as a cytoplasmic protein with physiologic functions such as regulating synaptic vesicles, altering synaptic plasticity, and serving as a molecular chaperone (Zhang et al., 2017b). However, abnormal $\alpha$-synuclein is implicated in numerous degenerative diseases, including PD (Allen Reish and Standaert, 2015). Many studies have provided evidence showing that $\alpha$-synuclein could exacerbate neuronal death, as it inhibits tubulin polymerization (Chen et al., 2007), interacts with membranes (Pacheco et al., 2015), and augments oxidative stress (Gao et al., 2008). Moreover, aggregated $\alpha$-synuclein could activate the inflammatory response by triggering the inflammasome and producing radicals (Gao et al., 2008; Codolo et al., 2013). In addition, modifications of $\alpha$-synuclein, especially nitrative $\alpha$-synuclein, could also potentiate neuron degeneration (Gao et al., 2008). The toxicity of nitrated $\alpha$-synuclein involves the following (Gao et al., 2008; Schildknecht et al., 2013): inhibiting both its own degradation and that of other proteins, decreasing $\alpha$-synuclein binding to membranes, stabilizing the monomer and inhibiting its fibrillization, and decreasing the clearance of $\alpha$-synuclein. Whether the phosphorylation of $\alpha$-synuclein at Ser129 is toxic remains unclear, but it is highly associated with PD because, among the total $\alpha$-synuclein deposited in LBs, approximately $90 \%$ is phosphorylated at Ser129, whereas only $\leq 4 \%$ is phosphorylated in the normal brain (Xu et al., 2015). The levels of Ser129-phosphorylated $\alpha$-synuclein differ between patients with PD and healthy individuals in both blood plasma and the cerebrospinal fluid, indicating its role as a useful biomarker for PD (Wang et al., 2012). Furthermore, the prion-like behavior of $\alpha$-synuclein is closely associated with the progression of PD (Sacino et al., 2013). All in all, $\alpha$-synuclein is a promising target for developing therapeutic strategies against neurodegenerative diseases. In our study, MPTP/p led to an increase in $\alpha$-synuclein as well as its aggregation, nitration, and phosphorylation, and $20 \mathrm{C}$ administration could reduce the levels of $\alpha$-synuclein and suppress its aggregation and nitration. 20C was unable to decrease phosphorylated $\alpha$-synuclein levels; therefore, its action is unrelated to the phosphorylation of $\alpha$-synuclein.

Another key factor for PD-related degeneration is neuroinflammation, which is closely affiliated with BBB permeation and glial activation. The brain had been ever taken as immune privileged because of its intact BBB structure, until peripheral immune cells were detected in the brain (Chen and Palmer, 2008; Brochard et al., 2009). Furthermore, the brain has its own resident immune cells (microglia), which comprise $20 \%$ of glial cells in the central nervous system (CNS) and survey the brain for injuries (Chen and Palmer, 2008; Maguire-Zeiss and Federoff, 2010). Both the BBB and microglia have key roles in the development and progression of neuroinflammation, which is involved in the degeneration observed in PD (Chen and Palmer, 2008; Chung et al., 2016). BBB damage can lead to the recruitment of $\mathrm{T}$ lymphocytes to the CNS and cause an inflammatory response by inducing microglial activation (Chen and Palmer, 2008; Chung et al., 2016), increasing the production of inflammatory factors such as tumor necrosis 


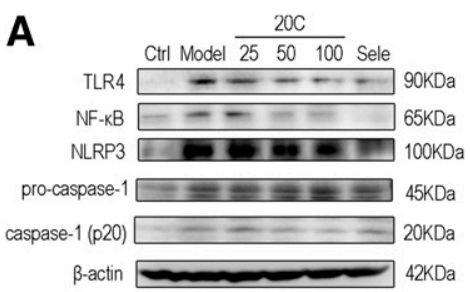

E

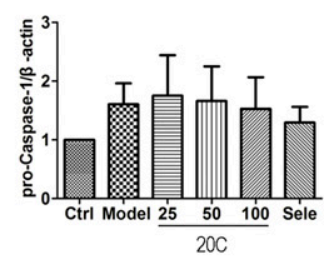

H

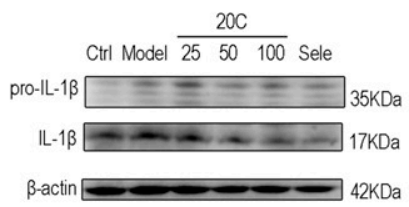

B

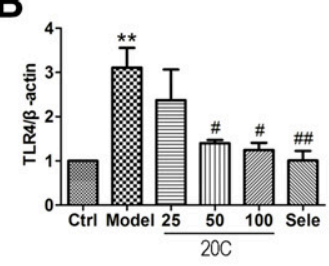

C

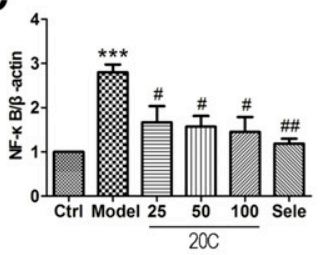

D

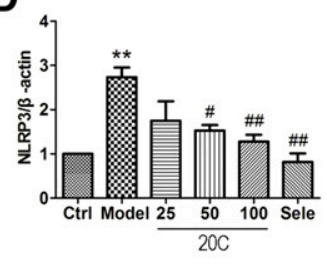

F

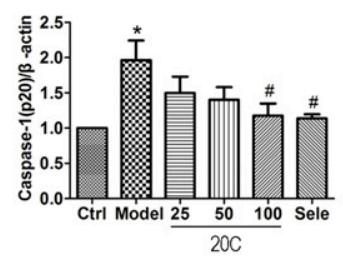

I
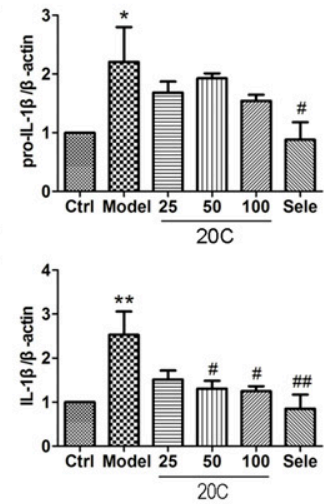

G

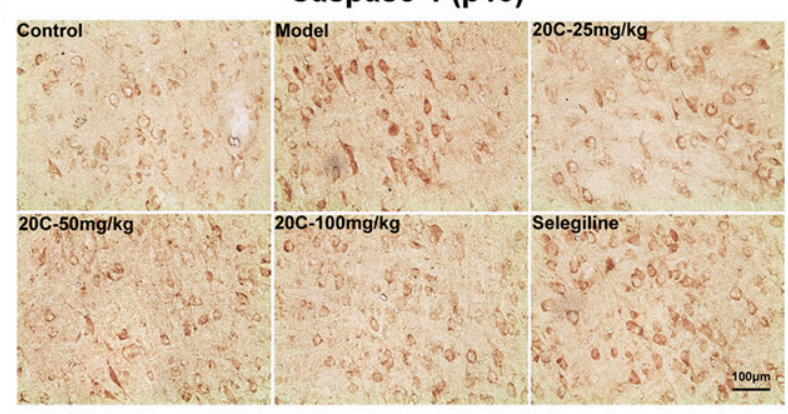

K

IL-1 $\beta$

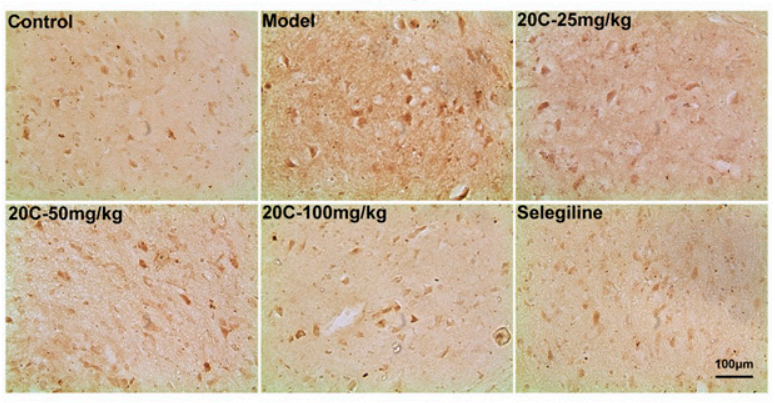

M

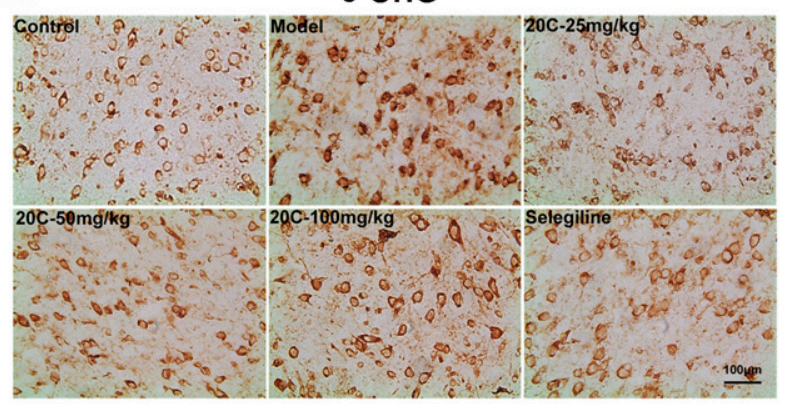

\section{8-OHG}

L

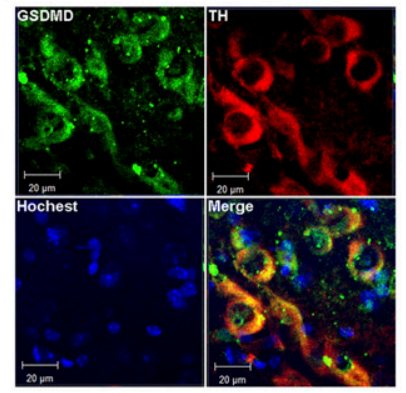

$\mathbf{N}$
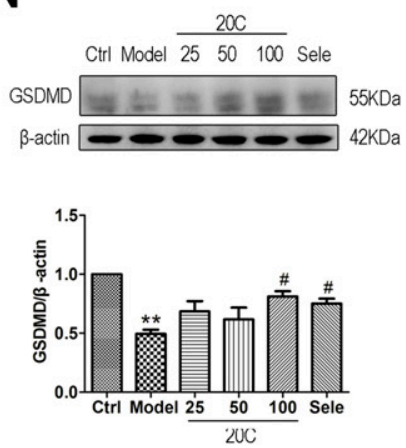

Fig. 5. Regulation of 20C on MPTP-induced NF- $\kappa$ B signaling and the NLRP3 inflammasome pathway. (A) Western blot bands of TLR4, NF- $\kappa$ B, NLRP3, and caspase-1 (pro- and p20). (B-F) Western blot analysis of TLR4, NF- $\kappa$ B, NLRP3, and caspase-1 (pro- and p20). (G) Immunohistochemical results for caspase-1 (p10). (H-K) Western blot and immunohistochemical results for IL-1 $\beta$. (L) Laser scanning confocal results for GSDMD and TH. (M) Immunohistochemical results for 8-hydroxyguanosine. (N) Western blot results for $55 \mathrm{kDa}$ GSDMD. Data are presented as means \pm S.E.M. $(n=3)$. Oneway analysis of variance was followed by Duncan's post hoc tests. ${ }^{*} P<0.05 ;{ }^{*} P<0.01 ;{ }^{* * *} P<0.001$ vs. the control group; ${ }^{\#} P<0.05 ;{ }^{\# \#} P<0.01$ vs. the model group. Ctrl, control; Sele, selegiline.

factor- $\alpha$ and IL- $1 \beta$. These toxicity mediators can in turn aggravate BBB damage (Reale et al., 2009). In addition to $\mathrm{BBB}$ and microglial cells, astrocytes are also widely accepted as pathologic hallmarks in progressive diseases. On one hand, similar to microglia, astrocytes can trigger the release of proinflammatory factors (Halliday and Stevens, 2011). On the other hand, astrocytes can induce microglial activation to further accelerate the progression of neuroinflammation (Fellner et al., 2011; Halliday and Stevens, 2011; Barbierato et al., 2013; Facci et al., 2014). Both BBB leakage and glial activation have been discovered in clinical and in in vivo studies (Reale et al., 2009; Halliday and Stevens, 2011; Chung et al., 2016). In this study, we found BBB injury with severe swelling of the astrocytic end-feet, which are largely activated microglia that became more amoeboidic by losing their ramifications, and activated astrocytes with enlarged bodies. $20 \mathrm{C}$ exhibited a protective effect against these abnormal alterations. 
A

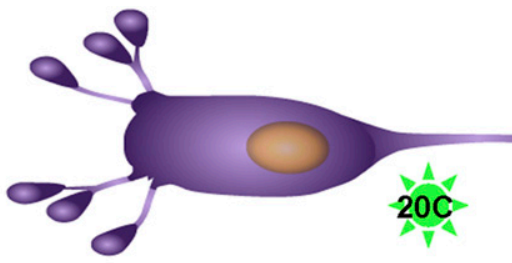

dopaminergic neuron damadge

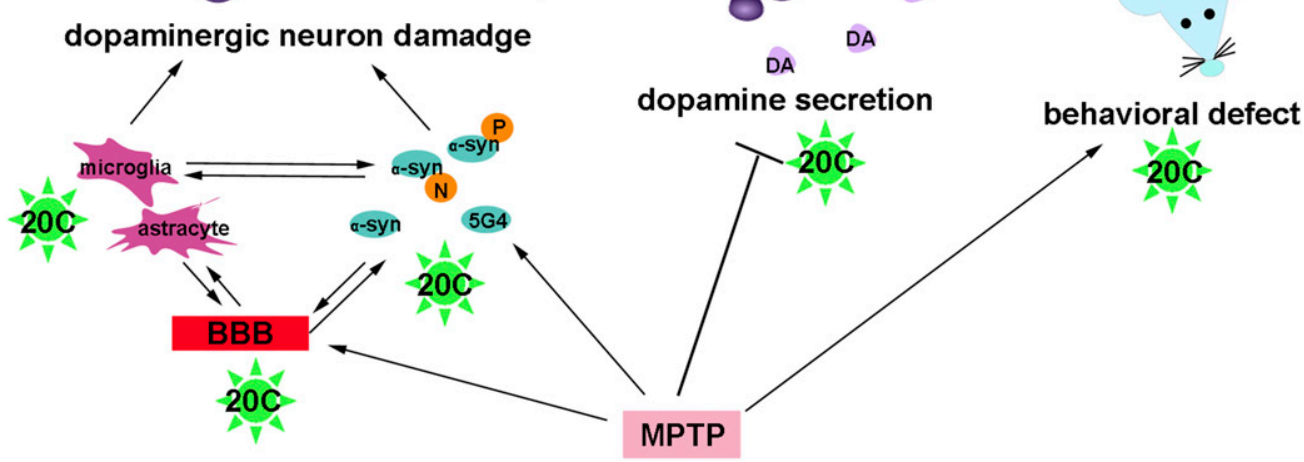

B

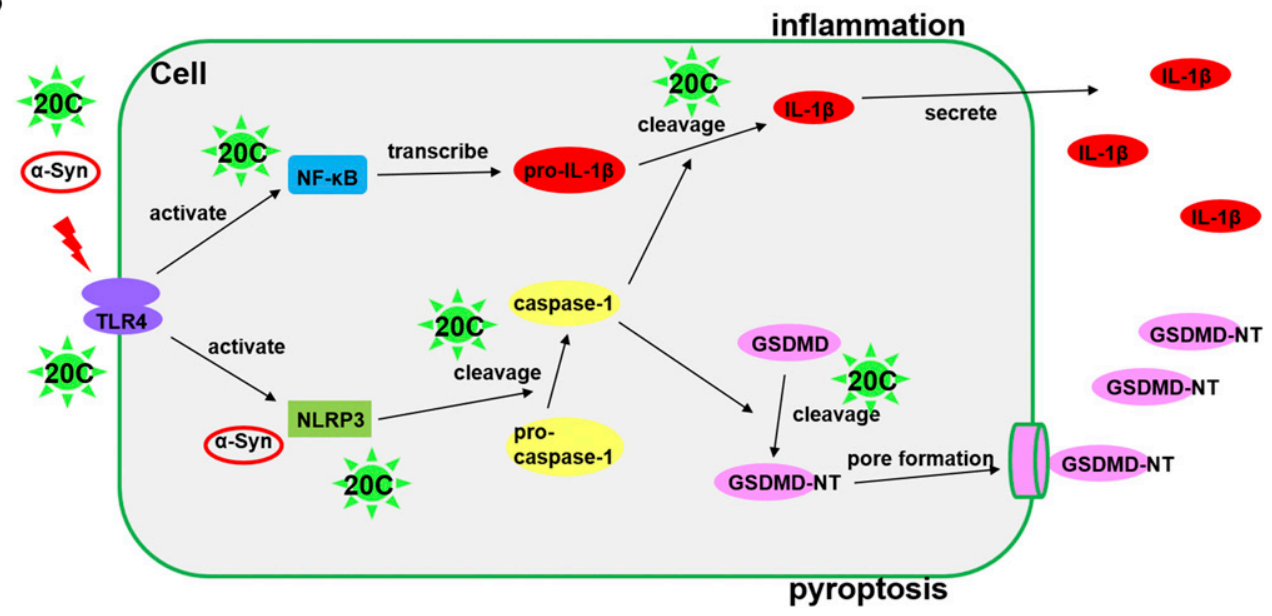

Fig. 6. Summary of this research. (A) $20 \mathrm{C}$ could prevent progressive neurodegeneration by interrupting the deteriorative cycle formed by BBB damage, glial activation, and $\alpha$-synuclein abnormality. (B) $20 \mathrm{C}$ could inhibit the activation of the NF- $\kappa$ B pathway and NLRP3 inflammasome, reducing both IL- $1 \beta$ release and inflammatory cell death (pyroptosis). NT, N terminus.
In fact, two factors for PD-related degeneration ( $\alpha$-synuclein dysfunction and neuroinflammation) can aggravate each other's toxicity by forming a deteriorative cycle. Pathologic $\alpha$-synuclein can induce neuroinflammation and oxidative stress by glial activation, and neuroinflammation can further cause the aggregation, nitration, and phosphorylation of $\alpha$-synuclein. Moreover, $\alpha$-synuclein can enhance BBB permeability (Jangula and Murphy, 2013) which can induce innate immunity and inflammation in the brain. 20C may disrupt the vicious circle and prevent the progression of $\mathrm{PD}$ by targeting $\alpha$-synuclein (Fig. 6A).

The molecular mechanisms of neuroinflammation mainly involve NF- $\kappa \mathrm{B}$ signaling and the inflammasome pathway (Fig. 6B) (Afonina et al., 2017). NF- $\kappa$ B signaling can be induced by $\alpha$-synuclein through triggering the pattern recognition receptor TLR4 (Zhang et al., 2017b). After NF- $\kappa$ B activation, inflammatory factors such as IL- $1 \beta$ will be largely produced (Zhang et al., 2017b). Inflammasome formation requires a cytosolic pattern recognition receptor as a sensor, such as NLRP1, NLRP3, NLRP6, NLRP7, NLRP12, NLRP4, and the absent in melanoma-2 (AIM2) (de Rivero Vaccari et al., 2014). Among these inflammasomes, the NLRP3 inflammasome has been the most investigated (de Rivero
Vaccari et al., 2014). NLRP3 can be primed by TLR ligands and then trigger downstream caspase-1 signals (He et al., 2016). The activated caspase- 1 is responsible for both the maturation of IL- $1 \beta$ from pro-IL- $1 \beta$ and the release of the GSDMD N terminus (Aglietti and Dueber, 2017). Mature IL- $1 \beta$ has a neurotoxic effect on the CNS, whereas the released GSDMD $\mathrm{N}$ terminus can form pores on the cell membrane by binding lipids to drive cell pyroptosis (Aglietti and Dueber, 2017). Furthermore, pore formation in pyroptosis can accelerate IL- $1 \beta$ release from dying cells (Wright and Bryant, 2016), and activated IL- $1 \beta$ can promote NLRP3 expression, which would in turn accelerate the inflammasome pathway (He et al., 2016). In this study, MPTP/p treatment led to an increase in TLR4, NF- $\kappa$ B, NLRP3, caspase- 1 , and IL- $1 \beta$, and a decrease in GSDMD (55 kDa); therefore, $\alpha$-synuclein-related $\mathrm{NF}-\kappa \mathrm{B}$ signaling and NLRP3 inflammasome pathway activation may be involved in neuronal loss after MPTP/p treatment. $20 \mathrm{C}$ could regulate all of these molecular changes.

In this study, we demonstrated the efficacy of $20 \mathrm{C}$ in vivo and proposed that $20 \mathrm{C}$ plays a protective role by reducing pathologic $\alpha$-synuclein and suppressing neuroinflammation. Furthermore, at the molecular mechanism level, 20C could inhibit activation of NF- $\kappa \mathrm{B}$ and the NLRP3 inflammasome 
pathway, reducing both IL-1 $\beta$ release and inflammatory cell death (pyroptosis).

\section{Acknowledgments}

We thank Zheng Mu, Peng-Fei Yang, Jv-Yang Huang, and CongYuan Xia for providing technical assistance.

\section{Authorship Contributions}

Participated in research design: Q.-S. Zhang, Yuan, N.-H. Chen.

Conducted experiments: Q.-S. Zhang, Heng, Y. Chen, Luo, Wen,

Z. Zhang.

Performed data analysis: Q.-S. Zhang.

Wrote or contributed to writing of the manuscript: Q.-S. Zhang.

\section{References}

Afonina IS, Zhong Z, Karin M, and Beyaert R (2017) Limiting inflammation-the negative regulation of NF-kappaB and the NLRP3 inflammasome. Nat Immunol 18:861-869.

Aglietti RA and Dueber EC (2017) Recent insights into the molecular mechanisms underlying pyroptosis and gasdermin family functions. Trends Immunol $\mathbf{3 8}$ 261-271.

Allen Reish HE and Standaert DG (2015) Role of $\alpha$-synuclein in inducing innate and adaptive immunity in Parkinson disease. J Parkinsons Dis 5:1-19.

Barbierato M, Facci L, Argentini C, Marinelli C, Skaper SD, and Giusti P (2013) Astrocyte-microglia cooperation in the expression of a pro-inflammatory phenotype. CNS Neurol Disord Drug Targets 12:608-618.

Bové J and Perier C (2012) Neurotoxin-based models of Parkinson's disease. Neuroscience 211:51-76.

Brochard V, Combadière B, Prigent A, Laouar Y, Perrin A, Beray-Berthat V, Bonduelle $O$, Alvarez-Fischer D, Callebert J, Launay JM, et al. (2009) Infiltration of CD4+ lymphocytes into the brain contributes to neurodegeneration in a mouse model of Parkinson disease. $J$ Clin Invest 119:182-192.

Chen L, Jin J, Davis J, Zhou Y, Wang Y, Liu J, Lockhart PJ, and Zhang J (2007) Oligomeric alpha-synuclein inhibits tubulin polymerization. Biochem Biophys Res Commun 356:548-553.

Chen Z and Palmer TD (2008) Cellular repair of CNS disorders: an immunological perspective. Hum Mol Genet 17 (R1):R84-R92.

Chung YC, Shin WH, Baek JY, Cho EJ, Baik HH, Kim SR, Won SY, and Jin BK (2016) CB2 receptor activation prevents glial-derived neurotoxic mediator production, BBB leakage and peripheral immune cell infiltration and rescues dopamine neurons in the MPTP model of Parkinson's disease. Exp Mol Med 48: e205.

Codolo G, Plotegher N, Pozzobon T, Brucale M, Tessari I, Bubacco L, and de Bernard $M(2013)$ Triggering of inflammasome by aggregated $\alpha$-synuclein, an inflammatory response in synucleinopathies. PLoS One 8:e55375.

Connolly BS and Lang AE (2014) Pharmacological treatment of Parkinson disease: a review. JAMA 311:1670-1683.

Dauer W and Przedborski S (2003) Parkinson's disease: mechanisms and models. Neuron 39:889-909.

de Rivero Vaccari JP, Dietrich WD, and Keane RW (2014) Activation and regulation of cellular inflammasomes: gaps in our knowledge for central nervous system in jury. J Cereb Blood Flow Metab 34:369-375.

Facci L, Barbierato M, Marinelli C, Argentini C, Skaper SD, and Giusti P (2014) Tolllike receptors $2,-3$ and -4 prime microglia but not astrocytes across central nervous system regions for ATP-dependent interleukin-1ß release. Sci Rep 4:6824.

Fellner L, Jellinger KA, Wenning GK, and Stefanova N (2011) Glial dysfunction in the pathogenesis of $\alpha$-synucleinopathies: emerging concepts. Acta Neuropathol 121: $675-693$.

Gao HM, Kotzbauer PT, Uryu K, Leight S, Trojanowski JQ, and Lee VM (2008 Neuroinflammation and oxidation/nitration of alpha-synuclein linked to dopaminergic neurodegeneration. J Neurosci 28:7687-7698.

Halliday GM and Stevens CH (2011) Glia: initiators and progressors of pathology in Parkinson's disease. Mov Disord 26:6-17.
He Y, Hara H, and Núñez G (2016) Mechanism and regulation of NLRP3 inflammasome activation. Trends Biochem Sci 41:1012-1021.

Heng Y, Zhang QS, Mu Z, Hu JF, Yuan YH, and Chen NH (2016) Ginsenoside Rg1 attenuates motor impairment and neuroinflammation in the MPTP-probenecidinduced parkinsonism mouse model by targeting $\alpha$-synuclein abnormalities in the substantia nigra. Toxicol Lett 243:7-21.

Huang JY, Yuan YH, Yan JQ, Wang YN, Chu SF, Zhu CG, Guo QL, Shi JG, and Chen $\mathrm{NH}$ (2016) 20C, a bibenzyl compound isolated from Gastrodia elata, protects PC12 cells against rotenone-induced apoptosis via activation of the Nrf2/ARE/HO-1 signaling pathway. Acta Pharmacol Sin 37:731-740.

Jangula A and Murphy EJ (2013) Lipopolysaccharide-induced blood brain barrier permeability is enhanced by alpha-synuclein expression. Neurosci Lett 551:23-27.

Liu LF, Song JX, Lu JH, Huang YY, Zeng Y, Chen LL, Durairajan SS, Han QB, and Li M (2015) Tianma Gouteng Yin, a traditional Chinese medicine decoction, exerts neuroprotective effects in animal and cellular models of Parkinson's disease. Sci Rep 5:16862.

Maguire-Zeiss KA and Federoff HJ (2010) Future directions for immune modulation in neurodegenerative disorders: focus on Parkinson's disease. J Neural Transm (Vienna) 117:1019-1025.

Mou Z, Yuan YH, Lou YX, Heng Y, Huang JY, Xia CY, Gao Y, Zhu CG, Chu SF, Luo $\mathrm{P}$, et al. (2016) Bibenzyl compound 20c protects against endoplasmic reticulum stress in tunicamycin-treated PC12 cells in vitro. Acta Pharmacol Sin 37: $1525-1533$

Ojemann LM, Nelson WL, Shin DS, Rowe AO, and Buchanan RA (2006) Tian ma, an ancient Chinese herb, offers new options for the treatment of epilepsy and other conditions. Epilepsy Behav 8:376-383.

Pacheco CR, Morales CN, Ramírez AE, Muñoz FJ, Gallegos SS, Caviedes PA, Aguayo LG, and Opazo CM (2015) Extracellular $\alpha$-synuclein alters synaptic transmission in brain neurons by perforating the neuronal plasma membrane. J Neurochem 132 : 731-741.

Reale M, Iarlori C, Thomas A, Gambi D, Perfetti B, Di Nicola M, and Onofrj M (2009) Peripheral cytokines profile in Parkinson's disease. Brain Behav Immun 23:55-63. Sacino AN, Brooks M, McGarvey NH, McKinney AB, Thomas MA, Levites Y, Ran Y, Golde TE, and Giasson BI (2013) Induction of CNS $\alpha$-synuclein pathology by fibrillar and non-amyloidogenic recombinant $\alpha$-synuclein. Acta Neuropathol Commun 1:38.

Schildknecht S, Gerding HR, Karreman C, Drescher M, Lashuel HA, Outeiro TF, Di Monte DA, and Leist M (2013) Oxidative and nitrative alpha-synuclein modifications and proteostatic stress: implications for disease mechanisms and interventions in synucleinopathies. $J$ Neurochem 125:491-511.

Wang Y, Shi M, Chung KA, Zabetian CP, Leverenz JB, Berg D, Srulijes K, Trojanowski JQ, Lee VM, Siderowf AD, et al. (2012) Phosphorylated $\alpha$-synuclein in Parkinson's disease. Sci Transl Med 4:121ra20.

Wright JA and Bryant CE (2016) The killer protein Gasdermin D. Cell Death Differ 23:1897-1898.

Xu Y, Deng Y, and Qing H (2015) The phosphorylation of $\alpha$-synuclein: development and implication for the mechanism and therapy of the Parkinson's disease. $J$ Neurochem 135:4-18.

Zhang QS, Heng Y, Mou Z, Huang JY, Yuan YH, and Chen NH (2017a) Reassessment of subacute MPTP-treated mice as animal model of Parkinson's disease. Acta Pharmacol Sin [published ahead of print].

Zhang QS, Heng Y, Yuan YH, and Chen NH (2017b) Pathological $\alpha$-synuclein exacerbates the progression of Parkinson's disease through microglial activation. Toxicol Lett 265:30-37.

Zhang XL, Yuan YH, Shao QH, Wang ZZ, Zhu CG, Shi JG, Ma KL, Yan X, and Chen NH (2017c) DJ-1 regulating PI3K-Nrf2 signaling plays a significant role in bibenzyl compound 20C-mediated neuroprotection against rotenoneinduced oxidative insult. Toxicol Lett 271:74-83.

Address correspondence to: Yu-He Yuan, Department of Pharmacology, Institute of Materia Medica, Chinese Academy of Medical Sciences and Peking Union Medical College, Xiannongtan Street, Xicheng District, Beijing 100050, P.R. China. E-mail: yuanyuhe@imm.ac.cn; or Nai-Hong Chen, Department of Pharmacology, Institute of Materia Medica, Chinese Academy of Medical Sciences and Peking Union Medical College, Xiannongtan Street, Xicheng District, Beijing 100050, P.R. China. E-mail: chennh@imm.ac.cn 